\title{
Introduction à l'étude de la saltation
}

\author{
Introduction to the study of saltation
}

\author{
PAR \\ P. DANEL \\ wl \\ R. DURAND EI E. CONDOLIOS

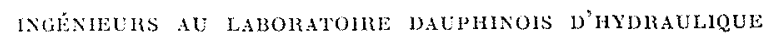

DIREGTEUR DU LABORATOHE DAUPHINOIS D'HYDRAULIQUE

\begin{abstract}
On a généralement sous-estimé le rôle important joué par la saltation dans les problemes d'entrainement de matérianx.

Les caractéristiques essentielles de ce mouvement sont : la perte de contact des grains abec « lancer vers le hant $\gg$ et les choes des grains all moment de leur relombée sur le dépôt de fond. Les " lancers vers le hant 》 sont favorisés par la modification des conditions d'éconlement autour des grains au moment de leur envol.

Au stade alu monbement généralisé, l'ensemble des particules se déplace a l'intérieur d'une « couche de saltation » parfailement délimitée qui parait conditionner la turbulence de l'écon-
\end{abstract} lement.

\begin{abstract}
The important role played by sallation in problems of movement of materials is generally ander-estimated.

The essential characteristics of this movement are : the particles' loss of contact as they are "thrown upwards" and the shocks as they fall back to the bottom deposit. The "throwing uplwards" is enconraged by modification of flow conditions round the particles at the moment they leave the bottom.

At the general stage of movement the group of particles moves to the interior of a well-defined "saltation layer" which appears to condition turbulence of the flow.
\end{abstract}

\section{INTRODUCTION}

On a généralement tendance à sous-estimer le ròle important joué par la saltation dans les problèmes d'entrainement de matériaux.

En effet, on considere d'ordinaire qu'il s'agit simplement d'un processus de transport intermediaire entre le glissement et la suspension. Le phénomène n'est généralement cité que pour mémoire. Certaines observations nous portent, tout au contraire, à penser que la saltation joue toujours un ròle essentiel dans le comportement des rivières à fond mobile, en particulier en ce qui concerne le modelé des fonds, la détermination des profils en travers et la pente tels qu'ils résultent de l'action réciproque des débits liquides et des matériaux de fond.

Certes, le terme saltation n'est pas nouveau dans les techniques qui nous occupent. A pre- miere vue, il pourrait sembler inutile de revenir sur les descriptions déjà données de ce mode de charriage des matériaux. Nous croyons tout au contraire devoir réexaminer avec soin le comportement des matériaux en saltation et analyser plus en détail les différents aspects de la nature physique du phénomène.

On s'étonnera peut-être qu'un tel phénomène qui, comme nous le montrerons, a déjà été si bien décrit, soit tombé dans un demi-oubli et qu'on n'ait pas cherché à l'intégrer dans les descriptions et théories du transport solide au même titre que, par exemple, les effets de la turbulence sur la suspension.

Il serait sans doute intéressant de rechercher attentivement les causes scientifiques ou psychiques de ce rang tout à fait sccondaire, voire épiso- 
dique, que l'on fait jouer d'habitude à ce qui fait l'objet du présent mémoire. Et pourquoi ne pas se demander tout d'abord pourquoi nous-mèmes, connaissant le phénomène, avons été si longtemps avant d'en apprécier toute l'importance et de chercher à mieux préciser ses traits fondamentaux.

Sans approfondir ce côté de la question, nous pouvons d'ailleurs déjà remarquer ici que si l'on se trouvait depuis longtemps devant une description quasi-définitive de certains aspects essentiels des phénomènes, ceux-ci n’avaient pas été réduits en formules et ne se prêtaient donc pas tels quels aux déductions mathématiques.

La turbulence, par contre, moins facile à observer et à décrire, a été l'objet de théories approchées, d'où l'on a pu déduire une description mathématique de la suspension turbulente qui, malgré des imperfections évidentes, a déjà rendu des services à l'ingénieur. Nous eroyons devoir affirmer toutefois qu'il nous semble nécessaire, pour faire progresser l'étude du charriage de fond et du transport en suspension, d'étudier attentivement la saltation qui est, selon nous, un facteur essentiel, si l'on ne veut pas voir les diverses théories du transport solide plafonner irrémédiablement.

Dans cette introduction à l'étude de la saltation, nous allons tout d'abord rappeler la description qualitative du phénomìne en reprenant tout d'abord les passages essentiels des mémoires fondamentaux de G. K. Gilbert et de R. A. BAGNOLD que nous avons choisis dans une vaste littérature comme étant de ceux qui ont décrit le plus minutieusement et le plus complètement la saltation.

A ces citations, nous joindrons les commentaires qui nous ont été suggérés par nos observations et nos mesures. Ensuite, nous reprendrons plus en détail le mécanisme des échanges de fond en ramenant l'ensemble des mouvements observés à un petil nombre de cas schématiques. Enfin, dans un chapitre sur la couche de saltation, nous aborderons l'étude slobale du phénomene

Mais comme l'indique le titre de ce mémoire, il ne s'agit encore ici que d'une introduction à une étude à laquelle nous travaillons depuis plusieurs années.

\section{CHAPITRE PREMIER}

\section{REMARQUES PRÉLIMINAIRES SUR LA SALTATION}

II est classique de distinguer dans les problimes d'entrainement des matériaux deux domaines distincts : le charriage de fond et le transport par suspension.

M. Bagnold illustre la nécessité de cette classification d'une manière originale :

«Après de nombreux vovages dans le désert, au cours descuels j'ai rencontré des tempêtes de sable d'intensité diverse, j'ai acquis la conviction que le mouvement du sable (opposé à celui de la poussière) est uniquement un phénomène de surface qui se localise dans une zone d'un mètre au-dessus du sol et que les tourbillons a large échelle n'ont aucun effet appréciable sur le transport des grains... Une conception populaire distingue d'ailleurs, dans les tempêtes de sable, la poussière du sable... Au début d'une tempête, le ciel s'obscurcit... mais après quelques heures, il ne subsiste plus qu'un nuage bas et dense dont l'épaisseur est au maximum de $2 \mathrm{~m}$. Cela st sensible par l'usure constatée sur les roches, qui devient inapprèciable à plus de $40 \mathrm{~cm}$ du sol... En dehors des régions accidentées, le nuage de sable glisse sur le désert en présentant l'aspect d'un tapis de haute laine. »

Prédominance de la Saltation

DANS LES PHÉNOMENIES DE CHARRIAGE DE FOND

Le terme de saltation semble avoir été utilisé pour la première fois par Mc Gée en 1908. L'expression est très imagée; il s'agit d'un mode de progression des grains par bonds successifs.

Parmi les trois processus que lon considere d'ordinaire en charriage de fond : glissement, roulement, saltation, le dernier est pratiquement le plus important.

G.-K. Grmbert :

« En charriage de fond, le glissement est un fac- 
teir négli̛geable. La rugosité de fond oblige les particules à rouler. Lorsque les grains sont de dimensions uniformes, chaque grain, pour se déplacer, doit franchir un obstacle de dimensions analogues aux siennes, et lorsqu'il atteint le sommet de cet obstacle, il possède d'ordinaire une vitesse suffisante pour sauter. Ainsi, le roulement n'est-il simplement qu'un prélude à la saltation. En charriage de fond, le mode de transport dominant est la saltation. »

Les fluctuations turbulentes semblent n'avoir dans la majorité des cas qu'un effet très secondaire sur les trajectoires des grains.

\section{G.-K. Gilbert :}

«Vue de la paroi, la saltation occupe sur le fond du courant une zone dont la limite supérieure est bien définic et parallèle au fond de sable. Dans cette zone, la distribution des grains «volants»est systématique, plus faible vers le haut que vers le bas, mais sans variation locale brusque perceptible. Vue du dessus, la surface du nuage semble uniformo et plane. Il est manifeste que le mouvement a lieu dans une même direction sans que cela suggèrc l'existence de tourbillons importants dans le courant... Cette variation continue des vitesses et des autres caractéristiques du mouvement que l'on observe sur tonte la hauteur du nuage ne cadre pas avec l'idée que la zone de saltation est soumise à l'influence de tourbillons de larges dimensions qui seraient capables de soutenir les grains. S'il existait des mouvements ascendants, la surface supérieure du nuage serait localement soulevée ef déprimée. Nous devons donc en déduire que l'écon. lement est turbulent, au sens technique du terme, mais que les tourbillons sont de faibles dimensions. $\gg$

Ceci est à rapprocher de la comparaison citée plus haut de M. BAGNold avec un « lapis de haute laine ».

MÉcaNisme DE DÉTAH DE LA SALTATHON.

\section{G.-K. GILbert :}

On constate que les grains semblent "projetés du fond avec une vitesse initiale, ce qui leur donne une trajectoire analogue à celle d'une balle de fusil $\gg$.

Gilbert propose comme explication le fait que le centre de gravité du grain décrit un are de cerele au cours du pivotement du grain sur l'obstacle placé à l'aval. Il apparaîtrait une force centrifuge qui favoriserait le décollement du grain.

\section{G.-K. Gillberti :}

«Si le roulement initial du grain sur le grain voisin est long, il a le temps d'acquérir de la vitesso avant d'effectuer son bond; la vitesse ascensionnelle est alors grande et l'angle d'ascension relativement fort. La trajectoire est en conséquence haute et longue. $\gg$

\section{R.-A. BAGNOLD :}

"Pour expliquer le mouvement des grains de sable en saltation, on peut dire " qu'ils se meuvent a la facon des balles de «ping-pong ». Ayant reçu de l'énergie du vent, ils acquièrent une vitesso horizontale qui se convertit, sous l'effet de la gravité, en vitesse d'impact. Si le sol est recouvert de grains durs et si les balles sont élastiques et résilientes, elles rebondissent vers le haut. Même si les balles tombent sur des balles identiques, elles penvent former à la surface du dépôt un petit cratère en éjectant d'autres balles, si bien que l'énergie qu'elles ont aecquise n'est pas entièrement perdue. 》

Le mouvement de progression des grains peut, en définitive, se décomposer en un mouvement d'oscillation vertical et un mouvement général d'entraînement par le fluide. L'énergie nécessaire pour entretenir le mouvement d'oscillation vertical est prélevée sur le fluide dans la partie ascendante de la trajectoire des grains. L'influence de la gravité sur le grain incurve ensuite la trajectoire et transforme la vitesse horizontale du grain en vitesse d'impact. suivant un processus remarquablement décrit par GiLiber't.

Nos observations nous ont en outre montré (que, pour les transports solides importants, Jes couches supérieures en saltation entrainent, par leur choe sur les couches infóricures, le glissement force de ces dernieres. Ainsi le glissement apparaît ici de facon paradoxale comme une conséquence de la saltation. Nous reviendrons de facon plus délaillée sur ce phènomène dans le chapitre suivant.

Après cet apercu général sur le phénomène de saltation, au cours duquel nous avons eu l'occasion de faire de larges emprunts à Grubert et à BAGNoLD, nous allons maintenant regarder plus en détail le mécanisme des échanges de fond. 


\section{MÉCANISME DES ÉCHANGES DE FOND}

Dans le chapitre précédent, nous avons cherché à donner un aperçu des idées de nos devanciers sur la saltation. Il nous parait bon de reprendre maintenant d'une façon plus ordonnée la deseription du mécanisme de détail du mouvement de saltation au voisinage du fond.

Nous étudierons successivement la maniere dont les grains bondissent littéralement depuis le fond, les effets de leur chute sur le fond, le glissement des couches de fond provoqué par le véritable bombardemeent qu'elles subissent, enfin l'allure générale des trajectoires de saltation.

\section{I. - Description du bond initial des grains}

Le début du mouvement d'un grain est étroitement lié à sa forme et à la position particulière qu'il occupe sur le fond. Nous examinerons d'abord le comportement de grains de formes plus ou moins régulières $\left({ }^{*}\right)$ et de dimensions sensiblement identiques. Potir définir les principales positions caractéristiques d'un grain au début de l'entrainement, nous serons amenés à schématiser les differentes positions relatives des grains. Nous nous inspiuerons pour eela de l'étude de Blancher pour les barrages en pierres lancées. Sans doute Brancmer avait-il essentiellement pour objet l'étude de l'arrêt des pierres en mouvement dans l'eau, alors que nous étudions ici leur mise en mouvement et l'entretien de ce mouvement. Les deux problèmes sont d'ailleurs étroitement liés, si bien que nos observations nous amènent à considérer schématiquement les mèmes positions principales que celles qu’il a utilisées.

\section{A. - Positrons schématroves DE DÉBUT D'ENTRAINEMLNT POUR DES GRAINS RÉGULIERS.}

La figure 1 définit quatre positions principales pour des grains de formes plus ou moins arrondies reposant sur un lit constitué par des particules sensiblement de même grosseur.

Position $n^{\circ} 1$. - Le grain repose isolément

(*) En ce qui concerne la définition de la forme des frains, nous nous référerons à la communication de R. DURAND, Mémoires et Travaux, Volume $\mathrm{I}-1951$.

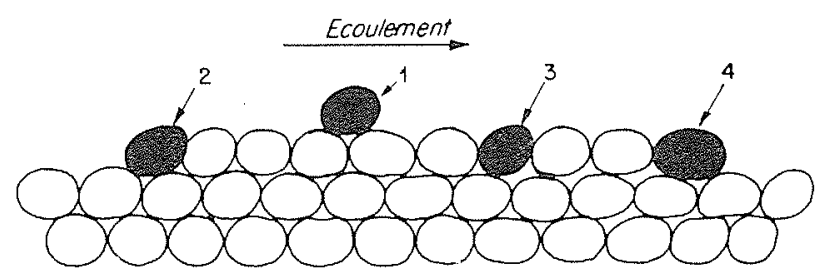

Fic. 1. - Positions schématiques pour les grains réguliers.

sur un lit sensiblement régulier et uniforme constitué par des particules de haute taille.

Position $n^{\circ}$ 2. - Le grain repose, comme le précédent, sur un lit régulier, mais s'appuie à l'aval sur une couche de grains disposés comme lui-même sur une autre couche de grains analogues.

Position $n^{\circ} 3$. - Le srain fait partie d'une couche relativement régulière qui constitue la surface du lit directement en contact avec le courant.

Position $n^{\circ}$ 4. - Le grain est à l'aval et à l'abri d'un grain analogue, mais n'est pas mieux bute que dans la position $n^{\circ} 1$.

$$
\text { B. - Conditrons dE DÉPART }
$$

POUR LES DIVERSES POSITIONS SCHÉMATIQUES.

On concoit aisément que les conditions de départ seront nettement différentes pour des grains placés respectivement dans les positions schématiques que nous venons d'envisager. La position (2) qui, pour Branchet, était la position clef pour la formation des massifs en pierres lancées, l'est egalement pour l'étude de la saltation. Nous montrerons en effet que les grains qui prennent leur départ en position 1,3 et 4 , n'effectuent de véritables bonds que Iorsque, entrainés par le courant, ils se retrouvent tout à coup en position (2). C'est donc cette dernière que nous étudierons en premier.

\section{DEPART EN POSITION (2).}

1. - Equilibre du grain au moment du départ. - Les conditions dans lesquelles se trouve placé le grain en équilibre immédiatement avant le début du mouvement ont été très souvent décrites, en particulier par IsBach et BLANCHET. Sui- 
vant le schéma classique présenté par la plupart des auteurs, il est alors soumis à deux forces principales (fig. 2):

- Le poids apparent du grain $\mathrm{P}_{a}$;

- La résultante des forces hydrodynamiques $\mathrm{T}$, généralement supposée proportionnelle au

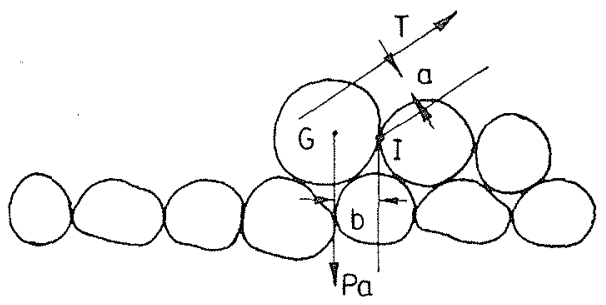

Fis. 2. -..- Position initiale et equilibre des forces.

carré de la vitesse et à un coefficient de forme et de position.

Il est commode pour notre probleme de décomposer la résultante des forces hydrodynamiques en une force verticale et une force horizontale qui, par certains côtés, rappellent la portance et la trainée d'une aile d'avion.

Pour une particule sphérique placée sur une surface plane et soumise à l'influence d'un écoulement, la composante verticale représente environ $25 \%$ de la composante horizontale proprement dite. Ce résultat a été établi en particulier par les expériences de Lossmevsy et d'ABriMov. L'existence d'une telle composante verticale est aisée à concevoir. Elle résulte d'une part de la courbure des filets sur le dessus de la sphere, ce qui augmente les vitesses et diminue considérablement les pressions locales, et d'autre part d'une différence importante des pressions entre la moitié inférieure et la moitié supérieure du grain, tant à l'amont qu'à l'aval.

La résultante des forces hydrodynamiques qui s'exercent sur le grain sera donc généralement dirigée vers le haut et inclinée vers l'aval. Nous ne reprendrons pas le détail de l'étude de l'équilibre au départ, si souvent étudié. On voit sur la figure 2 le rôle joué par la disposition relative du centre de gravité du grain $(\mathbf{G})$, du point de contact de butée (I) et du point d'application de la résultante des forces hydrodynamiques. De toute façon, si l'on augmente graduellement les vitesses, il arrivera un moment où les difrérentes forces seront en équilibre et où le grain sera prêt à partir.

Remarquons que s'il est intéressant d'étudier cet équilibre au départ qui conditionne la mise en mouvement, on risquerait néanmoins d'avoir une opinion erronée sur le mouvement lui-même si l'on n'étudiait pas attentivement comment varie la résultante des forces hydrodynamiques dans les premières phases du mouvement. En effet, si ces forces variaient peu a partir de la position d'équilibre, le mouvement des grains ne serait probablement qu'un glissement ou qu'un roulement sur le fond à partir du moment où le grain échappe à sa butée. Or, tout au contraire, le grain se trouve souvent lancé vers le haut.

2. Phase iniliale du mouvement jusqu'd la «perte de contact». - On a parfois considéré que le grain décolle du fond sous l'effet des fluctuations turbulentes. Nous réservant de revenir plus join et plus en détail sur l'influence de la lurbulence que nous avons volontairement négligée jusqu'ici, nous signalerons dès à présent que si ses effets peuvent être sensibles sur le tout début du mouvement, elle ne permet pas cependant d'expliquer pourquoi le grain est éjecté du fond à la manière d'un projectile; les ordres de grandeur n'y sont pas.

Nous proposerons ici une explication de ce phénomene de bond. Dès que les forces hydrodynamiques dépassent de peu la valeur eorrespondant à l'épuilibre, te grain commence son mouvement ct change de ce fait de position. Il en résulte une modification locale de l'écoulement et, par suite, de la valeur de la résistance hydrodynamique elle-même. Simutanément les positions relatives du centre de gravité et du point d'appui se trouvent modifiées ainsi que la valeur de la réaction d'appui. Il peut arriver que ces nouvelles conditions soient moins favorables au mouvement ef le grain retombe. Mais il peut se produire, tout au contraire, un accroissement extrêmement rapide des forces hydrodynamiques et en particulier de la composante verticale, an cours de la phase initiale du mourement de roulement ou de giissement jusqu'à la perte du contact d'appui. C'est alors qu'aura lieu le départ en projectile, si caractéristique de la saltation.
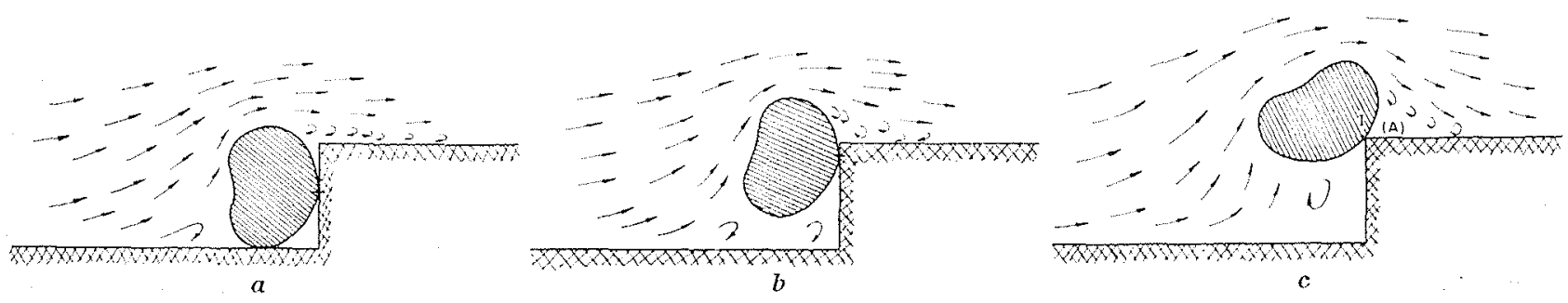

Fis. 3. - Passage de la position d'équilibre iniliale à la position de perte de contact. 
Pour sehématiser simplement notre explicaltion, nous considérerons un grain dallure sphérique placé initialement dans la position de la figure 3 a. Il commencera à se mettre en mouvement Iorsque la composante verticale deviendra quelque peu supérieure à la valeur de son poids apparent. Dès que le grain se soulere, la valeur de cette composante verticale augmente rapidement. Ceci résulte d'une part d'un accroissement des courbures de l'écoulement au-dessus du grain, entraînant une dépression sur la partie supérieure. D'autre part, si dans la position initiale la base du grain était soumise vers l'amont à une pression supérieure à celle de l'écoulement général et de l'ordre de la charge hydrodynamique, dès que le grain se soulève, ces pressions intéressent une surface nettement plus grande du grain.

Lorsque le grain arrive dans la position «perte de contact » (voir fig. $3 c$ ), non seulement il peut être soumis à une composante verticale plusieurs fois supéricure à celle qui avait suffi à lui faire quitter sa position d'équilibre initiai, mais encore il a déjà acquis une vitesse importante sous l'effet des forces hydrodynamiques graduellement et rapidement variables auxcruelles il a été soumis depuis son départ.

Il se trouve alors lancé vers le haut et anorce une trajectoire de saltation.

Il faut remarquer ici que si le mouvement vers le haul du grain, depuis sa position de déparl jusqu'à sa position de « perte de contact $»$, est suffisamment rapide, les changements de la couche limite: sillage à l'aval du grain, rouleau à l'amont vers le fond, n'ont pas le temps de se développer au même rythme et le grain se comporte alors presque comme s'il se trouvait dans un fluide parfait. Cette absence totale de sillage à l'aval recrée une zone de pressions dans la région (A), alors que dans les parties supérieures la courbure du grain crée des dépressions importantes puisque momentanément l'écoulement contourne le grain sans décoller. De ce fait, la résultante des forces hydrodynamiques peut se redresser vers la verticale. Dans quelques cas particuliers, la composante horizontale pourrait même être dirigce vers l'arrière. C'est là, crovonsnous, une des explications possibles de l'existence de trajectoires verticales ou même légèrement di. rigées vers l'arrière que l'on observe parfois. Après l'envol, graduellement mais assez rapidement, le sillage normal s'établit ainsi que la résistance hydrodynamique correspondante et te grain s'accélère dans le sens du courant.

lors du passage de la position ( $3 a$ ) à une position telle que $(3 b)$, le mouvement du grain peut être une combinaison de roulement et de glissement sur l'appui, landis que la phase finale de " perte de contact » s'accompagne générale- ment d'une rolation rapide aulour du point de contact (I). D'alleurs, nous n'avions parlé jusqu'ici pour simplifier que d'une resistance hydrodynamique alors que le plus souvent le grain sera solumis à la fois a laction d'une force et d'un couple, ce qui peut favoriser le mouvement de rotation du grain sur sa butée. G.-K. Grubsert a voulu voir, dans le mouvement du grain au moment de sa perte de contact, la raison principale de « l'envol» des grains, mettant en cause l'influence possible d'une force centrifuge résultant de cette rotation. Cette explication nous semble insuffisante. On pourrait dire également que cette rotation provoque un «effet Marves comme on le sait, donne une légère composante verticale qui contribue à la montée du grain, mais là encore il ne s'agit, nous semble-t-il, que d'un effet secondaire, bien que certain.

Nous croyons donc que la cause principale de Ia saltation doit être recherchée, comme nous venons de le voir, dans l'augmentation rapide des forces hydrodynamiques agissant sur le grain depuis sa position de départ jusqu'à la «perte de contact».

\section{INFLIENCE DE LA TYRBULENCE.}

Pour simplifier l'exposé, nous n'avons pas discuté du rôle de la turbulence sur les conditions d'équilibre au départ du grain et pendant la phase initiale du mouvement. Pour schématiser linfluence des fluctuations turbulentes sur ce mouvement, nous remarquerons tout d'abord que seuls les tourbillons plus gros que les grains considérés pourront avoir un effet sensible sur ces grains; de même, les fluctuations de vitesse n'interviendront que si elles présentent une durée suffisante, compte tenu de l'inertie des grains.

Soit un grain en équilibre de départ pour une vitesse thériquement égale à $V_{0}$ en l'absence de toute turbulence. Ce grain pourra prendre le départ en mouvement turbulent pour une valeur de la vitesse locale moyenne $V_{1}$ inférieure à $V_{0}$ si les fluctuations de vitesse atteignent une valeur $\Delta V_{1}$ telle que $V_{1}+\Delta V_{1}=V_{0}$.

Si l'écart $\Delta V_{I}$ que nous venons de considérer est un maximum, le grain n'aura pas le temps d'amorcer son mouvement et retombera immédiatement, c'est-à-dire qu'à l'observation on le verra en quelque sorte « frémir » ou « palpiter $\gg$ de temps à autre. Si au contraire l'écart $\Delta V_{1}$ considéré dure un temps suffisant, le grain se soulèvera assez pour que, bénéficiant de l'augmentation de la résultante hydrodynamique déja signalée, il puisse continuer son mouvement, même si la vitesse est retombée par exemple à une valeur voisine de $V_{1}$. On voit donc, sans 
insister davantage, qu'il y aurait lieu de considérer ici une valeur efficace de la fluctuation de vitesse suffisante pour assurer ce mourement et telle que :

$$
\mathrm{V}_{1}+\Delta \mathrm{V}_{10 \mathrm{ft}}=\mathrm{V}_{0}
$$

Finalement, si la turbulence change quelque peu les conditions de départ, elle change peu les ordres de grandeur des vitesses nécessaires pour entraîner le mouvement des grains. Elle explique, par contre, la plupart des "frémissements» des grains qui s'observent fréquemment et que l'on ne pourrait pas toujours expliquer autrement. Nous reviendrons plus loin sur l'influence de la turbulence après « l'envol» du grain.

Signalons enfin que la fluctuation de vitesse, que nous avons considérée ici comme une caractéristique de la turbulence, pourrait tout aussi bien être l'effet, dans une conduite, d'une onde de coup de bélier, ou résulter dans un canal découvert d'une onde ou d'une intumescence engendrant sur le fond une fiuctuation de vitesse jouant un rôle analogue.

Pour résumer le mouvement d'un grain, initialement placé en position (2), sur le début de sa trajectoire, nous avons tracé à titre d'exemple la figure 4.

\section{C. - Départ d'un grain régulier} A PARTIR DES AUTRES POSITIONS.

Position $n^{\circ}$ 1. - En position (1), le grain ćtant bile tout en sautant d'un grain à l'autre. Comme il perd constamment le contact, on pourrait déjà considérer ce mouvement comme une saltation, bien qu'il n'y ait pas encore les lancers vers le haut si caractéristiques de la saltation.

Le fond étant loin d'être régulier, le grain subira une serie de chees plus ou moins forts el rencontrera de véritables obstacles qu'il franchira plus ou moins facilement. En schématisant, on peut dire que tant qu'il restera au cours de son mouvement dans ce que nous appellerons la position (1), il roulera ‘n sautillant jusqu’à ce qu'il rencontre un obstacle capable de le ramener en position (2). C'est alors que «l'envol », tel gue nous l'avons déjà décrit, pourra se produire. Suivant les positions respectives du centre de gravité du grain et du point de choc, la vitesse initiale du grain pourra ou non aider son envol. Dans le cas où l'énergie cinétique facilite le mouvement, le grain s'élèvera pour des vitesses moyennes de l'écoulement inférieures à celles qui auraient été nécessaires s'il avait été buté au repos dans cette même position (2).

Ja figure 5 résume l'ensemble du mouvement pour le cas que nous venons de considérer.

Position $n^{\circ}$ 3. - En position (3), le grain ne démarrera le plus souvent que s'il se trouve ramené en position (2) par le dégagement des grains situés à l'amont ou si sa position est modifiće fortuitement, de façon directe ou indirecte, par le choc d'une particule en saltation.

Position $n^{\circ}$ 4. -...- En position (4), le grain ne

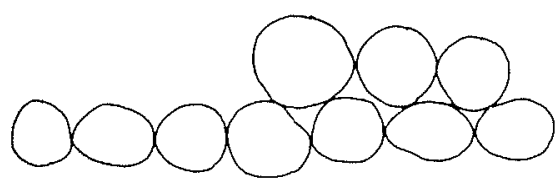

Frc. 4 a. - Grain en position d'équilibre initiale.

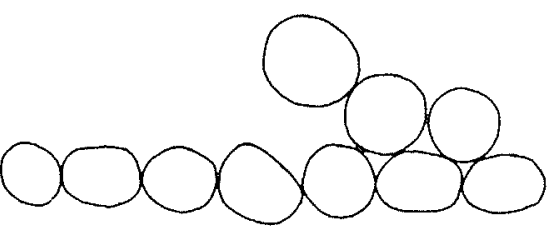

FIG. 4b. - Grain en position de perte de contact.

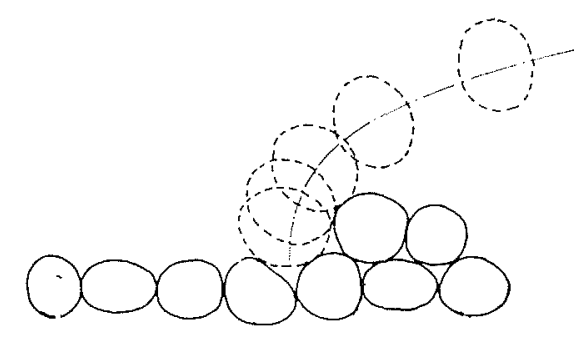

Fic. 4 c. - Accélération sur l'obstacle de brisée, décollement

et début de la trajectoire saltation. moins bien buté qu'en position (2) part plus facilement, c’est-à-dire en général pour une vitesse plus faible. Il pourra glisser, mais le plus souvent il roulera sur les grains de la couche immo- se metra en mouvement le plus souvent que sous l'effet direct ou indirect d'une autre particule en saltation. Il se retrouve alors sensiblement en position (1), (voir fig. 6). Bien entendu,

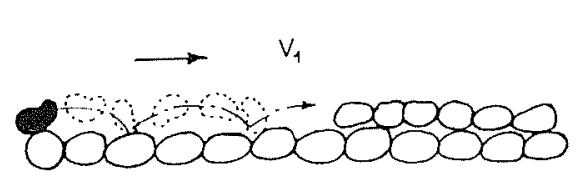

a. - Sautillement du grain.

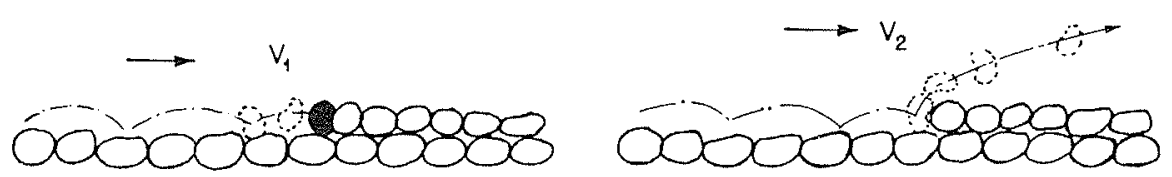

b. - Blocage des grains en position (2) c. - Départ en position (2) de butée. de butée.

Tia. 5. - Départ d'un grain arrondi en position (1). 


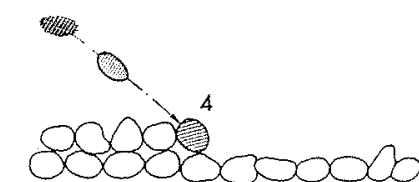

a. - Arrivée d’un projectile sur le grain en position (4).

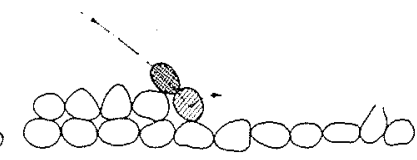

b. -..- Percussion.

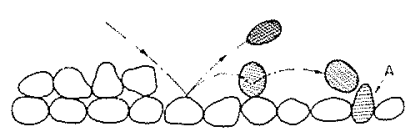

c. - Rebondissement du projectile et sautillement du grain (4).

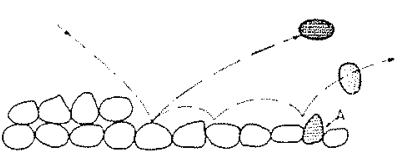

a. - « Envol 》 du grain (4) après réflection sur $A$.

Fig. 6. - Depart d'un grain arrondi en position (4).

il se retrouvera en position (1) si toute la couche qui le précède est emportée progressivement.

\section{D. -- DÉpart pOUR DES GRANS RELATIVEMENT PLATS \\ DE FORMES ET DE DIMENSIONS ANALOGUES.}

Dans cette courte introduction à l'étude du mécanisme de la saltation, nous avons considéré des grains que nous avons qualifiés de réguliers afin de schématiser un ensemble très complexe. Avant d'abandonner cette question et en nous inspirant toujours des schématisations de BLANCHET pour l'arrêt des pierres lancées dans l'eau, nous allons maintenant examiner brièvement le comportement de grains relativement plats, de formes et de dimensions analogues.

La figure 7 définit les quatre principales po-

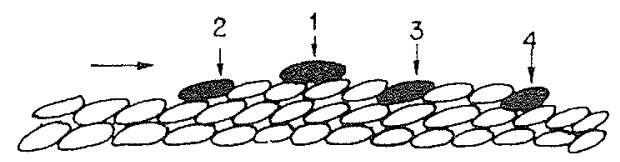

Fyc. 7. -.. Positions types

pour des pierres homogenes plates avec pendage vers l'amont.

sitions initiales d'équilibre pour des grains plats. Ces positions correspondent à celles définies par la figure 1 pour les grains de forme régulière.

re dépôt que nous considérons ici s'est constitué avec une tendance au pendage vers l'amont, ce qui est, comme l'a montré Brancher, le cas ginéral.
Le mécanisme de détail du départ des grains sera voisin de celui que nous avons déjà décrit. kemarquons cependant que lorsqu'un grain plat passe de la position d'équilibre initial à la posilion « perte de contact», l'augmentation brutale de portance est encore plus importante, du fait de la dissymétrie du grain.

Considérons maintenant le cas où le grain repose initialement sur un fond lisse (hg. $8 a$ ); il commencera par glisser el viendra heurter l'obstacle constitué par le grain marqué par la lettre d sur la figure.

Suivant la facon. dont la pierre se présentera au moment du choc, son comportement pourra être complètement different. Si l'avant du grain tend à se soulever, il se plaquera sur l'obstacle, adoptant lui aussi la position en pendage vers l'amont. Si c'est au contraire l'arrière du grain qui se souleve, le mouvement s'amplifiera et donnera naissance à une trajectoire de saltation suivant la figure $8 \mathrm{c}$.

\section{E. - REMaroues GÉNÉRALES SUR TE DEBBUT DU MOUVEMENT.}

Le début d'entrainement, au moment où les particules, initialement placées en position (1), sautent d'une aspérité à l'autre sans s'élever de facon vraiment appréciable, correspond au charriage de fond avec roulement et glissement, tel qu'il est décrit d'ordinaire par les auteurs. Pour nous, ce n'est là qu'une question de degré et il s'agit déjà d'une amoree de la saltation car on en retrouve dans ce début de mouvement cer. laines des caractéristiques essentielles a notre

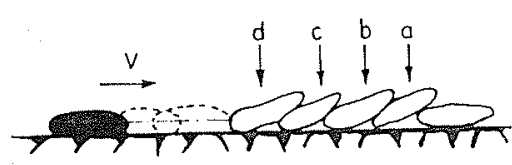

a. - Glissement sur le fond lisse.

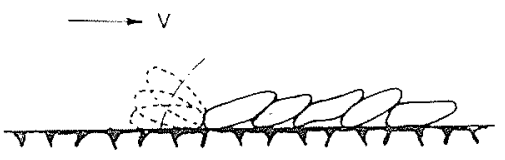

b. -- Pivotement sur 'l'obstacle de butée.

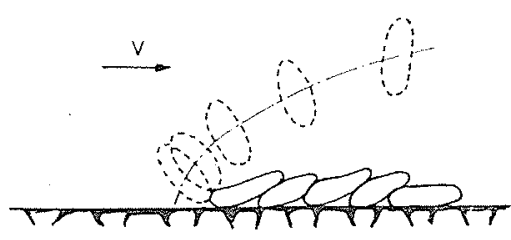

c. -... Début de la trajectoire de saltation.

Fug. 8. - Depart d'une pierre plate sur fond lisse. 
avis : perte de contact et chocs, sans cependant rencontrer les «lancers 》 vers le haut.

Remarquons encore que pour des vitesses mème un peu supérieures au début d'entraînement, la hauteur des bonds des grains est encore souvent inférieure à quelques diamètres. En observant l'écoulement à la verticale, on peut avoir l'impression d'un roulement ou d'un glissement. Par observation latérale, le phénomène de saltation se manifeste par contre de façon évidente.

\section{II. - Description de la retombée des grains}

Considérons un grain sur la fin de sa trajectoire de saltation au moment où il arrive en contact avec le dépôt. Il possède alors une vitesse importante qui lui a été communiquée par le fiuide, en particulier dans la partie supérieure de sa trajectoire.

Différents cas peuvent alor's se présenter :

1. -.- Le grain ricoche sur le haut d'une particule solidement contrebutée en position (3). Il rebondira à une vitesse assez voi-

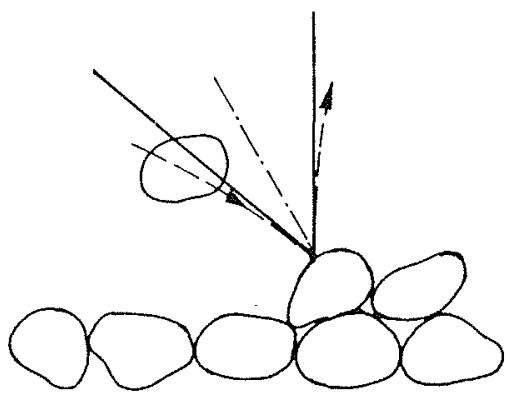

Fio. 9. - Réflection d'un grain sur la fin de sa trajectoire.

sine de sa vitesse initiale d'impact. Ce cas paraît bien être en fait assez exceptionnel, sauf aux faibles vitesses où le rendement des chocs demeurera satisfaisant (fig. 9).
2. --. Le grain ricoche sur des grains insulfisamment contrebutés. Il les mettra alors en mouvement en partageant avec cux son énergie cinétique.

La mise en mouvement de ces particules s'effectuera avec des pertes par frottement. Le choc proprement dit aux grandes vitesses entraînera également des ruptures des grains par éclats.

Dans la majorité des cas, le rendement du choc sera relativement mauvais et le grain ne rebondira, à proprement parler, que d'une hauteur assez faible. Pour s'en convaincre, il suffit de jeter une pierre avec violence sur un tas de pierres identiques. On n'observera qu'exceptionnellement un rebondissement franc.

Si le rendement du choc considéré du seul point de vue du grain est généralement faible, les conséquences pratiques de ce même choc sont par contre très importantes. Le départ des grains heurtés sera grandement facilité et le grain «primaire » conservera le plus souvent assez. d'énergie pour se retrouver en position de «perte de contact $"$, donc en mesure d'effectuer un nouveau bond. L'arrivée d'un grain sur un dépôt pourra donc provoquer l'amorce de plusieurs trajectoires de saltation (fig. 10). Le phénomène pourra s'amplifier jusqu'au moment où l'énergie disponible dans l'écoulement ne sera plus suffisante pour compenser les pertes et entretenir de nouveaux mouvements de saltation.

Ce principe de la multiplication des trajectoires de saltation que nous venons d'évoquer est illustré clairement par une expérience de R.-A. Bagnold.

Sur un dépôt de grains immobiles, avec une vitesse d'écoulement du fluide légèrement inférieure à la vitesse de début d'entraînement, il laisse tomber un mince filet de grains de sable. A la surface du dépôt et à l'aval de la zone d'impact, on constate un débit solide par saltation manifestement plus important que le débit solide injecté. Le fait qu'initialement les grains

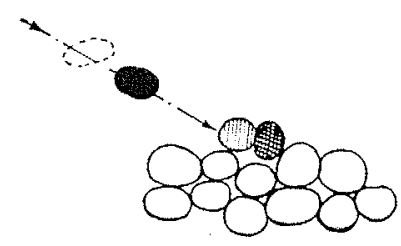

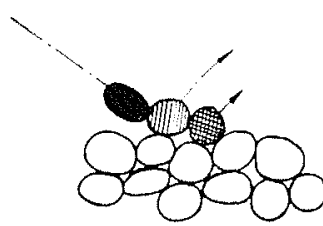

b

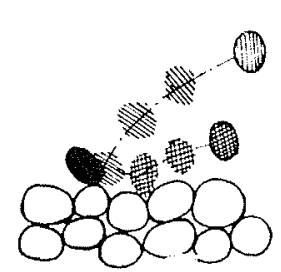

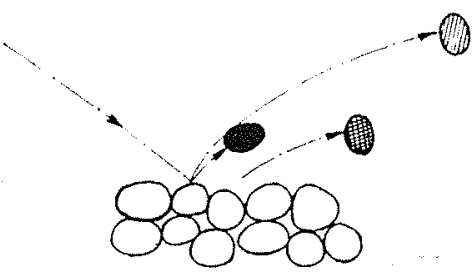

(1)

F1G. 10. - Multiplication des trajectoires de saltation. 
constituant le dépôt se trouvaient immobiles rend le phénomène particulièrement frappant. Il se reproduit évidemment de façon analogue si les particules de fond sont déjà en mouvement.

\section{III. - Glissement forcé des couches de fond}

Pour un mouvement de saltation suffisamment intense, nos observations nous ont permis de mettre en évidence l'existence d'un phénomène de glissement des couches de grains formant la surface du dépôt de fond.

Ce glissement est produit grâce aux composantes tangentielles fournies par les grains en mouvement de saltation au moment de leur percussion sur le dépôt.

L'énergie d'impact permet ainsi non seulement d'assurer la continuité du mouvement de saltation, mais encore entraîne le glissement des couches souls-jacentes (fig. 11).

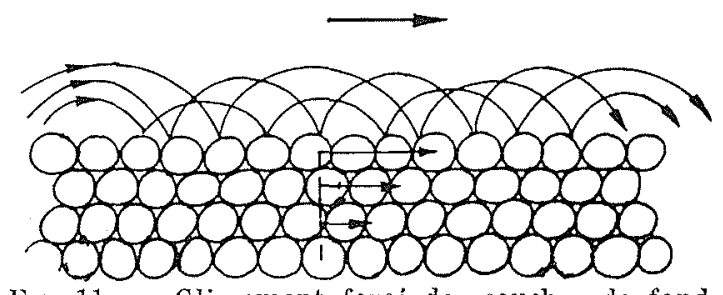

Fic. 11. - Glissement forcé des couches de fond.

Plusieurs couches supplémentaires peuvent ainsi être mises en mouvement. Leur vitesse de progression semble décroitre linéairement. Le glissement est évidemment gêné si les grains présentent un contour angulenx. Au contraire, si les formes sont suffisamment régulières el arrondies, on constate en même temps le roulement des grains les uns sur les autres. Ce phénomène de glissement n'est évidemment observable que par l'intermédiaire d'une paroi vitrée latérale. Il est totalement masqué par la couche de saltation si l'on observe l'écoulement par le dessus. Il est curieux de retrouver ici tres exactement le schéma imaginé par Du Boys, mais avec une interprétation physique différente. Le glissement, au lien d'être engendré directement par l'eau, l'est par l'intermédiaire du bombardement de la couche de saltation. Il ne s'agit plus d'un phénomène de début d'entraînement, mais au contraire d'un phénomène qui n'apparait que lorsque le débit solide acquiert une valeur appréciable.

Nous croyons devoir insister sur le fait que ce phénomène n'a rien de commun avec le phénomène exceptionnel du glissement d'un grain isolé sous l'action directe des forces hydrodynamiques.

\section{IV. - Saltation et usure}

Au début du mouvement des matériaux, lorsque la saltation commence, les chocs qui se produisent à la retombée des grains sur le dépot n'ont encore qu'une intensité réduite. Le choc sera le plus souvent purement élastique et les usures et ruptures en éclats seront peu fréquentes.

Par contre, lorsque le mouvement de saltation s'est généralisé, avec des grains suffisamment gros, les chocs répétés qui sont capables d'entraîner le glissement de plusieurs couches de dépòt de fond, amènent également une usure rapide des grains.

Pour des galets. on peut observer des ruptures par éclats (très fréquentes avec les silex), des brisures franches (particulièrement avec les calcaires) ou mème une désagrégation en sable pour les roches granitiques. On rencontre une forte proportion de galets présentant des traces de telles ruptures par exemple dans les bancs du Rhône à Donzère et dans ceux du Drac à Grenoble. Ces galets sont par ailleurs parfaitement polis et émoussés.

Si les grains sont de nature minéralogique différente, la retombée des grains durs sur le fond pourra amener une usure rapide des grains plus tendres. La nature réalise en quelque sorte un broyeur à boulets. Dans ce cas, les grains tendres ont une vie très courte et sont réduits en farine dès que le débit est suffisant pour mettre en route les graviers et les galets de roche dure.

D'ailleurs, dans une rivière, les graviers el les galets ne seront souvent mis en mouvement que pour des crues exceptionnelles, si bien que le rythme des usures dépendra de celui des crues. La forme même des galets sera en relation avee la loi de variation des débits, comme nous avions eu l'occasion de le signaler en 1950 dans la discussion d'une communication de M. TRICART.

Lorsque les grains sont devenus suffisamment petits, leur usure tend à devenir négligeable. En effet, leur vitesse de chute devient faible et les chocs diminuent d'intensité. Pour une sphère de diamètre donné, constituée par une roche d'élasticité donnée, on peut calculer la vitesse nécessaire pour que, dans la zone d'impact, la limite élastique se trouve dépassée et qu'apparaisse corrélativement une amorce d'usure. Pour de la silice, des vitesses d'impact de plusieurs mètres/seconde sont nécessaires pour des grains de quelques dixièmes de millimètre. Aussi, pour une rivière déterminée où les vitesses d'écoulement ne dépassent pas une certaine valeur maximum, on peut considérer que pour les sables quartzeux l'usure cesse pratiquement en dessous 
d'un certain diamètre de grains. Les sables les plus gros s'émoussent et s'arrondissent, mais les grains les plus fins conservent leurs arêtes vives comme on peut s'en assurer au microscope. Reprenons maintenant ce sable fin et placons-le dans un écoulement nettement plus rapide; les usures commenceront.

L'usure des sables des plages sous l'action de la mer pose des problèmes analogues.

\section{V. - Allure générale des trajectoires de saltation}

L'énergie cinétique ascensionnelle du grain, acquise par le grain dans sa position de «perte de contact $》$, représente une valeur appréciable, ce qui conduit à des trajectoires de saltation dont la hauteur est de l'ordre de plusieurs fois le diamètre du grain. Cette composante d'énergie cinétique ascendante se transforme en énergie de position au cours du mouvement de montée du grain. Le freinage hydrodynamique qui se produit est alors compensé par le fait que le grain pénètre dans une zone où les vitesses d'écoulement sont plus grandes. Le fluide communique au grain un supplément de vitesse horizontale qui fait qu'il rejoint le fond sur la fin de sa trajectoire avec une vitesse absoluc d'impact au moins du même ordre de grandeur que sa vitesse initiale de départ (sinon supéricure) (fig. 12).

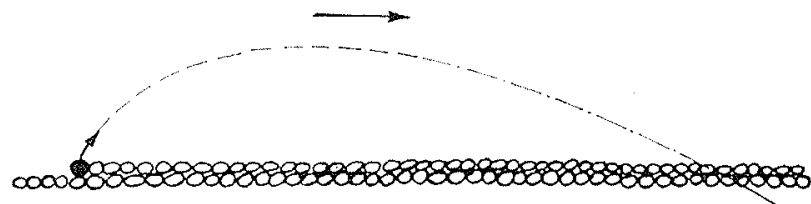

Fic. 12.- Allure générale d'une trajectoire.

Dans la partie ascendante de sa trajectoire, le srain possède une vitesse horizontale inférieure à la vitesse locale de l'écoulement tandis que sur la partie descendante il posside une vitesse supérieure.

La hauteur des trajectoires croit d'abord rapidement avec la vitesse, puis plus lentement:
Elle semble dépendre de la vitesse de chute des grains. Cetle dernière remarque est bien en accord avec le fait que la hauteur des trajectoires des grains, de l'ordre de plusieurs centaines de diametres dans l'air, n'est plus que de quelques dizaines de diamètres dans l'eau.

On peut donc considérer que le mouvement des grains, qui résulte en moyenne de la superposition d'un mouvement oscillant vertical et d'un mouvement d'entrainement général horizontal, a une vitesse inférieure mais assez voisine de la vitesse moyenne de l'écoulement.

Remarquons encore que les grains continuent a tourner sur eux-mêmes le long de Ja trajectoire. Ce mouvement de rotation est particulièrement intense dans l'air, ce que montrait clairement un film présenté par $M$. $Z_{\text {ING }}$ au Congrès d'Alger des Zones Arides en 1951. Par suite de cette rotation, le grain prend successivement toute une série d'orientations différentes par rapport à l'ecoulement et par suite sa résistance hydrodynamique ne correspond plus à une orientation déterminée du grain. Il faut plutòt considérer une moyenne. Cette remarque nous explique peut-être pourquoi l'influence de la forme particulière des grains tend à s'atténuer dès que la saltation devient assez intense, ce que nous avons pu vérifier par nos observations et nos mesures.

Nous arrêterons là nos descriptions du mécanisme de détail du comportement des grains dans le mouvement de saltation, bien que nous n'ayons encore discuté que de l'entrainement de grains de dimensions sensiblement analogues.

Lorsque la majorité des grains en mouvement suivent des trajectoires de saltation analogues à celles que nous venons de décrire et que la suspension pour les grains de dimensions similaires est un phénomène pratiquement négligeable, nous dirons avoir affaire à de la saltation à l'état pur.

L'extension de notre étude à l'analyse du comportement des mélanges nous entraînerait beaucoup trop loin et fera l'objet d'un autre mémoire.

Dans le chapitre suivant, nous examinerons par contre brièvement l'influence de la grosseur des grains supposés de mêmes dimensions sur l’allure du phénoméne de saltation.

\section{CHAPITRE III}

\section{LA COUCHE DE SALTATION}

Au cours de nos nombreuses observations sur le charriage par saltation, nous avons été souvent frappés de la valeur des premières observations de nos devanciers, tels que G.-T. GIL-
BERT et R.-A. BAGNold. Mais nous avons été surpris, par contre, que l'on ait si peu cherché $\dot{a}$ tirer parti de leurs remarquables travaux pour mieux comprendre non seulement les phénomè- 
nes classiques de charriage de fond, mais aussi ceux de la suspension qui semblent intimement liés en profondeur au phénomène de saltation.

Pour des vitesses moyennes un peu supérieures à celles correspondant au début du mouvement général, on constate en effet que le mouvement de saltation occupe une zone bien délimitée et présentant des caractérïstiques particulières. Nous proposons d'appeler cette zone « la couche de saltation $»$.

\section{LA « COUCHE DE SALTATION ».}

Lorsque la saltation est le phénomène dominant, la couche de saltation apparaît limitée supérieurement par une surface plane assez nette. Sur cette surface de séparation, on n'observe pas les volutes ou les déchirements caractéristiques d'une influence prépondérante de la turbulence à grande échelle de l'écoulement.

L'aspect général d'une couche de saltation à l'état pur est schématisé par la figure 13. Les

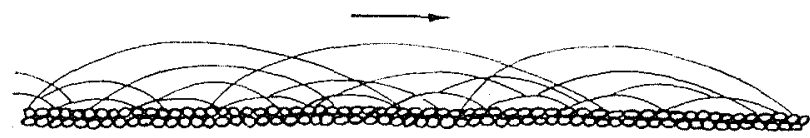

Fic. 13. - Allure générale des trajectoires dans une couche de saltation.

trajectoires présentent pratiquement une hauteur maximum. Les échanges d'énergie au moment de l'impact sur le fond, tels que nous les avons décrits dans le chapitre précédent, expliquent l'existence d'une telle limitation de l'importance des trajectoires. II est également évident que les grains ne se trouvent pas tous au moment de leur départ dans des conditions aussi favorables, et qu'en conséquence, ils effectuent des trajectoires dont la hauteur varie entre quelques diamètres et la hauteur maximum

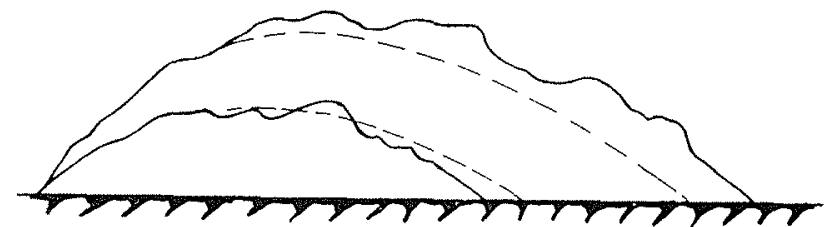

Fia. 14 a. - Modulation des tajactoires de saltation pour les composantes des fluctuations turbulentes.

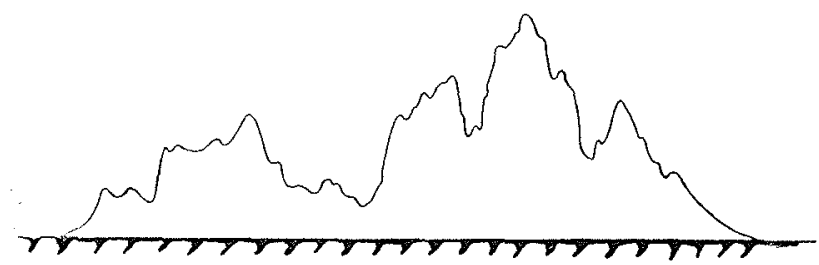

Fsa. 14 b. - Trajectoire du grain en suspension avec départ et arrivée en saltation. que nous venons de signaler. Les trajectoires de faible hauteur sont d'ailleurs les plus nombreuses.

Les fluctuations turbulentes peuvent prendre de l'importance si l'on considère des particules dont la vitesse de chute présente une valeur relativement faible. On constate alors une modulation désordonnée de la trajectoire, dont l'allure générale peut se trouver conservée (fig. 14a).

Enfin, si les fluctuations turbulentes comportent des composantes verticales de vitesse supérieures à la vitesse de chute des grains, ceuxci peuvent passer en suspension (fig. $14 \mathrm{~b}$ ). Mais, même dans ce cas, le départ des grains et leur retombée sur le fond se produisent toujours par saltation, a l'intérieur de ce que nous appellerons encore la « couche de saltation».

Cette dernière présente donc une individualité marquée, analogue à celle de la couche laminaire dans les écoulements turbulents lisses.

Nous allons maintenant exposer quelques remarques générales sur le comportement des grains vis-à-vis des tourbillons de l'écoulement, ce qui nous permettra de préciser ensuite, dans le cas des icoulements à surface libre en canal rectiligne, pour quelles dimensions de grains on observe la saltation pure ou la combinaison de la saltation et de la suspension turbulente.

\section{REMARQUES GṔNÉRALES}

SUR LA SUSPENSION ET LA SALTATION

Considérons un grain solide dans un fluide en mouvement. Parler de suspension revient en fait à considérer le comportement des grains visà-vis des mouvements d'agitation du fluide ef plus particulièrement de la turbulence.

D’une façon générale, quelle que soit la grosseur de la particule considérée, on peut toujours imaginer que l'écoulement comporte des tourbillons d'échelle et d'intensité suffisamment grandes pour que les fluctuations de vitesse verticale soient supérieures à la vitesse de chute des grains et présentent une durée suffisante pour agir sur ces derniers. Pour que la mise en suspension soit appréciable, il faut que les tourbillons soient nettement plus grands que les grains.

A chaque type d'écoulement correspond en fait une sorte de limite maximum d'échelle el d'intensité des tourbillons. Ainsi, pour chaque type d'écoulement, il pourra exister des grains trop gros pour pouvoir être mis en suspension.

Il serait intéressant d'étudier les conditions do similitude de la saltation et d'en préciser les limites. En première approximation, la saltation pure telle que nous l'avons décrite suit sensiblement la similitude de Froudr.

Par contre, lorsque les grains deviennent suf- 
fisamment petits, les phénomènes de cohésion et de plasticité viennent moditier le comportement relatif des grains et il n'est plus possible de parler de saltation.

Pour schématiser, nous présenterons une clas. sification des matériaux suivant leur dimension en fonction de leur possibilité de mise en mouvement par des écoulements-types simples.

Les colloïdes de dimensions inférieures au micron peuvent être maintenus en suspension sous l'effet du mouvement brownien du liquide, même dans un liquide rigoureusement immobile à notre échelle. Les argiles et les vases décantent lentement dans une eau parfaitement calme, mais une très faible turbulence suffit à assurer leur mise en suspension, par exemple la simple agiLation créée par une baguette dans un récipient. La même agitation restera pratiquement sans effet durable sur des sables fins ou des limons (30 y. à $0,15 \mathrm{~mm}$ ). Pour assurer leur mise en suspension, une turbulence convenablement entretenue est nécessaire, comme par exemple celle résultant $\mathrm{du}$ frottement de l'eau sur les parois d'une conduite ou d'un canal. Pour les sables grossiers, les graviers et les galets, la turbulence d'un écoulement naturel n'est plus toujours suffisante pour assurer leur mise en suspension. Ces matériaux progresseront donc le plus souvent par saltation dans une conduite ou dans une rivière. Pour assurer leur mise en suspension, il faudra laire appel à des effets turbulents plus intenses, comme ceux crés par un ressaut, un déferlement, ele... Ce sera par exemple le cas des galets dans les marmites des torrents. Citons également l'influence de fortes rugosités accidentelles sur les berges d'une rivière. Des grains de sable progressant nettement par saltation sur la pente douce constituée par le front amont d'une dune peuvent tournoyer indéfiniment dans le rouleau qui se développe à l'aval de la dune.

On peut réaliser un écoulement tel qu'il soit capable de transporter par saltation soit un sable de $0,5 \mathrm{~mm}$, soit des graviers de $20 \mathrm{~mm}$. Par contre, si on cherche à faire entraîner un mélange de ces deux matériaux par le même écoulement que précédemment, on constatera que manifestement le sable fin se trouve mis en suspension dans la zone de saltation des galets. La turbulence de sillage de ces derniers est alors d'échelle suffisante pour entraîner le sable dans un mouvement désordonné.

La classification très schématique des matériaux que nous venons d'exposer est en partie celle que nous avions déjà présentée pour les transports en conduite. Cette classification n'a ividemment rien d'absolu et n'est valable que pour la gamme d'écoulement où elle a été définie. Elle semble s'appliquer à peu près directement aux écoulements dans les canaux avec une légère diminution des dimensions critiques correspondant à la fin de la suspension pure et au début de la saltation pure. Pour le transport du sable par le vent, BAgNoli donne la valeur de $10 \mathrm{mi-}$ crons pour le diametre critique correspondant au passage de la suspension à la saltation.

Ia transposition de ce diamètre critique du cas de l'air au cas de l'eau peut se faire par des considérations de nombre de Reyolus associé au grain.

Pour que la saltation se développe nettement dans l'eau, on peut dire que pratiquement les grains devront être assez gros pour pouvoir ètre considérés comme indépendants les uns des autres. Ce sont les grains « incohérents » de l'école anglo-indienne.

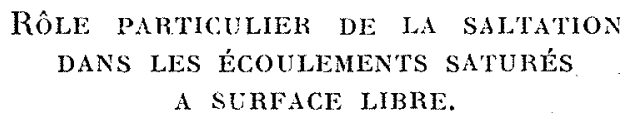

Si le matériau de fond est suffisamment fin pour être doué de cohésion, et si, pour une cause fortuite, cette cohésion vient à ètre rompue, les grains sont immédiatement mis en suspension et ne se déposent plus tant que l'écoulement conserve une vitesse suffisante. La turbulence normale d'un écoulement à surface libre suffit à assurer une répartition pratique homogène de la concentration. Pour une granulométrie uniforme, un tel matériau doit avoir un diamètre inféricur a 50 microns. Nous sommes alors dans un domaine très particulier, celui des matériaux doués de cohésion.

Dans un canal d'essais à parois latérales lisses, avec des grains ayant plus de quelques dixièmes de $m m$ de diamètre, on réalise par contre très facilement un transport par saltation pure. La limite supérieure de la « couche de saltation $\gg$ est très nette.

Pour des dimensions intermédiaires, le transport a lieu à la fois par suspension et par saltation. Mais tant qu'il subsiste un dépòt sur le fond, les grains progressent par bonds au ras du dépôt et l'on constate toujours l'existence d'une saltation pure près du fond.

\section{INTERACTION DE L'ÉCOULEMENT ET DE LA COUCHE DE SALTATION.}

Nous aborderons maintenant un nouvel aspect du problème : l'influence du mode de transport des grains sur la valeur des pertes de charge.

Il semble bien que le transport des matériaux par suspension, s'il a souvent une grande importance pratique an point de vue cubage d'allu- 
vions transportées, n'ait qu'une influence relativement négligeable sur les pertes de charge de l'écoulement ou sur la pente de la rivière. Ceci est à rapprocher du fait expérimental observé en conduite, suivant lequel, en suspension totale, les pentes de la ligne de charge sont les mêmes qu'en eau claire pour des sables fins (pour les argiles, la densité de mixture intervient directement comme s'il s'agissait d'un fluide homogène de densité différente de celle de l'eau). Par analogie, dans les rivières, les produits très fins fourniraient d'eux-mêmes, par leur énergie de position, le travail nécessaire à leur transport.

On peut dire finalement que l'entrainement des matériaux n'influerait sur la pente de la rivière de façon sensible que s'il y a saltation. On peut ainsi définir un "régime de saltation ». La couche de saltation, qui constitue une zone à part, agit finalement de façon analogue à une rugosité. Elle régit la turbulence de l'écoulement qui régit à son tour l'intensité de la suspension. Il est d'ailleurs à remarquer que s'il existe un grand nombre de formules reliant la pente au débit solide pour le charriage de fond, aucune formule de ce genre n'a jamais été établie pour la suspension.

Notre but, dans ce premier mémoire, nétail pas de montrer comment se fait la transition entre la couche de saltation et la suspension qui la surmonte parfois. Nous dirons cependant qu'il nous semble difficile, à la lumière de nos observations, de poursuivre efficacement l'étude de la suspension entretenue par la turbulence (austauch) sans mieux connaitre tout d'abord ce qui se passe dans la couche de saltation.

On pourrait enfin nous objecter que dans notre description du mouvement des grains nous avons négligé de parler des rides de fond. Cela nous aurait entraînés trop loin. Sur la surface d'une ride, la couche de saltation continue d'ailleurs d'exister et de jouer le rôle d'une rugosité régissant l'écoulement. La manière dont la couche de saltation se complique en présence des dunes fera l'objet d'un prochain mémoire.

\section{CONCLUSION}

En manière de conclusion, nous résumerons les principales caractéristiques de la saltation sous forme de propositions condensées.

1. - Le roulement sur le fond, sans qu'il y ait décollement franc ou simple perte de contact de grain, ne se concoit aisément et ne se constate que pour un fond sensiblement lisse et pour des particules d'allure sphérique ou cylindrique.

2. - Le glissement des grains sous l'action directe du fluide ambiant n'est possible que pour un fond assez lisse et pour certains cailloux pratiquement plats et convenablement orientés.

3. - Au début de l'entrainement, les particules sautent de grain en grain sans s'élever de façon appréciable; c'est déjà une amorce de la saltation car on retrouve là deux de ses caractéristiques essentielles : pertes de contact et chocs.

4. - Un grain isolé présentant des arètes marquées pivotera autour de l'une de ces arêtes au moment de son départ et téndra ainsi à amorcer un bond ou un choc, ou les deux à la fois.

5. - Si le grain bute sur un obstacle constitué par un autre grain de dimensions analogues, il basculera le plus souvent pour s'échapper. Il en résultera une tendance à la saltation.

6. - Dans le charriage de fond, la saltation est le phénomène dominant.

7. - Les trajectoires de saltation pure rappellent celles des projectiles d'artillerie et n'ont pas l'allure désordonnée des mouvements domines par Ja turbulence.
8. - Les lancers de grains vers le haut semblent s'expliquer par l'augmentation rapide des forces hydrodynamiques en jeu depuis la rupture d'équilibre jusqu'à la perte de contact.

9. - Ce phénomène de laneer des grains vers le haut s'explique même sans turbulence. Celleci ne vient que modifier quelque peu les vitesses moyennes de l'écoulement nécessaires pour mettre les grains en mouvement.

10. - Au stade du mouvement généralisé, les particules ricochent sur le fond, ne perdant ainsi qu'une part de l'énergie cinétique empruntée au fluide.

11. - A ce stade de mouvement généralisé, l'ensemble des grains en saltation se trouve dans une couche bien déterminée que nous avons appelée «couche de saltation ».

12. - La hauteur de la couche de saltation varie tout d'abord rapidement avec la vitesse et ensuite plus lentement. Elle semble dépendre de la vitesse de chute des particules.

13. - Aux vitesses usuelles, la hauteur des trajectoires dans l'air est de Pordre de plusieurs centaines de fois le diametre des grains. Dans l'eau, elle ne dépasse pas quelques dizaines de diamètres.

14. -- Lorsque la vitesse augmente, les trajectoires sont plus tendues et les bonds s'allongent. res chocs sont plus violents et la quantité totale de matériaux mise en mouvement augmente.

15. - Pendant qu'ils parcourent leurs trajec- 
toires, les grains tournent sur eux-mêmes, plus rapidement d'ailleurs dans l'air que dans l'eau.

16. - Les grains tournant sur eux-mèmes sont soumis à un «effet Magnus » peu important mais cependant certain.

17. - Lorsque la saltation a atteint une intensité suffisante, elle entraine le glissement d'une ou de plusieurs couches de grains du dépôt proprement dit.

18. - Le glissement de ces couches de fond s'accompagne de roulement si les grains ont des formes suffisamment régulières et arrondies.

19. - Les usures des grains résultent en majeure partie de la saltation et principalement des chocs sur le fond.

20. -- La rapidité de l'usure dépend essentiellement de l'intensité du phénomène de saltation et par conséquent de l'importance des débits de la rivière. Le rythme de l'usure suit le rythme des crues.

21. -- Pour un cours d'eau donné, les sables quartzeux ne s'usent plus en dessous d'un certain diamètre du grain. Ce diamètre limite dé- pend essentiellement de l'intensité de la saltation et variera par conséquent d'une rivière à l'autre.

22. - La surface supérieure d'une couche de saltation pure est nettement marquée et sensiblement horizontale.

23. - La surface supérieure de cette couche n'a pas l'aspect moutonné des courants de densité.

24. - Lorsque l'intensité de la turbulence est telle que les fluctuations de vitesse verticales dépassent sensiblement la vitesse de chute des grains, le phénomène de suspension turbulente apparaît.

25. - Même lorsque la suspension turbulente est assez intense, on constate encore les sautillements, les lancers et les retombées caractéristiques de la saltation. On peut donc toujours considérer qu'il existe une « couche de saltation 》 près du fond.

26. - Avec des grains assez fins pour être doués de cohésion, il n'est plus possible de parler de saltation.

\section{BIBLIOGRAPHIF}

1. R. A. Bagnold. - The Physics of blown sand and desert dunes. Bethuen, Londres, 1941.

2. R. A. BagnolD. - British Journal of Applied Physics. Vol. n² 2, pp. 29-34, février 1951 .

3. C. Blanchet. - Formation et destruction par un courant d'eau de massifs en pierres. La Houille Blanche, mars 1946.

4. A. Cailleux. - Indice d'émoussé. Définition et première application. Extrait du C.R.S. de la Société Géologique de France, 10 novembre 1947, p. 251.

5. H. J. CASEY. - Uber geschiebebewegung. Berlin, 1935.

6. P. Danel. - A.I.R.H. Congrès de Stockholm, 1948.

7. P. Du Boys. - Le Rhône et les rivières à lit affouillable. Annales des Ponts et Chaussées, 1879.

8. R. Durand. - Différentes définitions techniques de la forme des galets. La Houille Blanche, numéro spécial $A, 1951$.

9. R. Durand. - Transport hydraulique des graviers et galets en conduite. La Houlle Blanche, numéro spécial $\mathrm{B}, 1951$.

10. R. Durand, E. Condolios. - Etude expérimentale du refoulement des matériaux en conduite, en parti- culier des produits de dragage et des schlamms. S.H.F., Journées de juin, 1952.

11. G. K. Gilzent. - The transport of debris by running water. U.S. Geological Survey. Professional Paper, $n^{\circ} 86,1914$.

12. C. V. Isbach. - Les barrages en pierres lancées dans l'eau courante. Annales de l'Institut Hydrotechnique U.R.S.S., tome XVII, 1935, p. 12.

13. H. Kramer. - Transactions of A.S.C.E., Vol. 100, 1935, pp. 798-838.

14. I. I. LÉvx. - La dynamique des cours d'eau. Moscou, 1948.

15. W. J. McGee. - Geol. Soc. America Bull. Vol. 19, p. 199,1908 .

16. A. Shrelns. - Anwendung der Aehnlichkeit Mecanik und der Turbulenzforschung am geschiebebewegung. Preussischen Versuch fur Wasserbau und Schiffbau. Berlin, 1936.

17. U.S.W.E.S. - Studies of river bed materials an their movement. Paper 17, 1935.

18. C. M. White. - The equilibrium of grains on the bed of a stream. Proceedings of the Royal Society of London, $\mathrm{n}^{\circ} 958$, Vol. 174, p. 322-338, 1940. 